\title{
Síntesis y evaluación de recubrimientos base fluoruro empleando fuentes alternativas al HF sobre la aleación Elektron 21 para la fabricación de implantes ortopédicos biodegradables
}

\author{
Synthesis and evaluation of fluoride-based coatings using \\ alternative sources of HF on the Elektron 21 magnesium \\ alloy for biodegradable orthopedic implants application
}

\section{Síntese e avaliação dos revestimentos base fluoreto utilizando fontes alternativas ao HF sobre a liga dos magnesio Elektron 21 para a fabricação de implantes ortopédicos biodegradáveis}

Leidy Andrea Rojas Flórez ${ }^{1}$; Henry Alberto Briceño Urbina²; Carlos Andrés Hernández Barrios²* Claudia Nieves Barrera ${ }^{1}$; Darío Yesid Peña Ballesteros ${ }^{2}$; Fernando Viejo Abrante ${ }^{1}$; Ana Emilse Coy Echeverria ${ }^{2}$

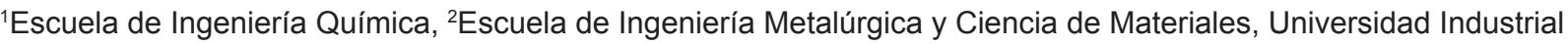
de Santander (UIS), Cra. 27 Calle 9, Bucaramanga, Colombia.

*carloshernandezbarrios@hotmail.com

Fecha Recepción: 15 de octubre de 2014

Fecha Aceptación: 09 de junio de 2015

\begin{abstract}
Resumen
En la actualidad se han postulado las aleaciones de magnesio como una alternativa prometedora en la fabricación de implantes biodegradables debido a su excelente degradabilidad, biocompatibilidad y propiedades mecánicas comparables a las del hueso; sin embargo, su elevada velocidad de corrosión hace necesario el diseño de recubrimientos biodegradables, entre los que se destaca aquellos sintetizados por conversión química en medio $\mathrm{HF}($ hasta $48 \% \mathrm{v})$. No obstante, el empleo de este ácido genera grandes problemas en términos de seguridad, ya que es altamente tóxico. Surge entonces la necesidad de hallar vías alternativas que permitan sustituir dicho ácido o limitar su uso a cantidades reducidas. En el presente trabajo se evaluaron mezclas $\mathrm{HF}(4 \% \mathrm{v})-\mathrm{NaF}$ y $\mathrm{H}_{3} \mathrm{PO}_{4}-\mathrm{NaF}$ como alternativas al empleo de HF en la síntesis de recubrimientos biodegradables sobre la aleación de magnesio Elektron 21. La caracterización microestructural de los recubrimientos se realizó mediante microscopía electrónica de barrido y difracción de rayos $\mathrm{X}$, mientras que la resistencia a la corrosión se evaluó mediante ensayos electroquímicos y gravimétricos en solución Hank a $27^{\circ} \mathrm{C}$. Los resultados mostraron que en presencia de $\mathrm{NaF}$, los recubrimientos sintetizados están constituidos por una bicapa $\mathrm{MgF}_{2-x}(\mathrm{OH})_{x} / \mathrm{NaMgF}_{3}$, donde la presencia de $\mathrm{NaMgF}_{3}$ evita el ataque localizado por picadura, haciendo que el proceso de degradación de los recubrimientos sea uniforme y progresivo. Particularmente, los recubrimientos sintetizados bajo la condición $\mathrm{H}_{3} \mathrm{PO}_{4} 1,6 \% \mathrm{v}-\mathrm{NaF}$ 0,5M mostraron un excelente comportamiento, superior a los obtenidos con el empleo de HF, por lo que se postulan como excelentes candidatos para el reemplazo inmediato de este ácido.
\end{abstract}

Palabras clave: recubrimientos, aleación, magnesio, implantes ortopédicos, biodegradable, corrosión. 


\begin{abstract}
Magnesium has been postulated as an excellent candidate for fabrication of biodegradable implants because of its degradability, biocompatibility and excellent mechanical properties comparable to bone's those. Nevertheless, its high corrosion rate represents a great disadvantage, allowing the approach of modifying its electrochemical behaviour by the design of biodegradable coatings. In this regard, the most common synthesis route employed is chemical conversion in HF (up to $48 \% \mathrm{v}$ ) to produce biodegradable magnesium fluoride coatings. However, it is well known that HF is an extremely hazardous acid. Thus, one of the main targets is to find other alternative routes to avoid its use or, at least, to reduce its concentration. In the present investigation $\mathrm{HF}(4 \% \mathrm{v})-\mathrm{NaF}$ and $\mathrm{H}_{3} \mathrm{PO}_{4}-\mathrm{NaF}$ solutions were evaluated as alternatives of the $\mathrm{HF}$ route to produce biodegradable coatings on the Elektron 21 magnesium alloy. Characterization of the conversion coatings was carried out by scanning electron microscopy and X-ray diffraction, whereas their corrosion resistance was evaluated by electrochemical and gravimetric measurements in Hank solution at $37^{\circ} \mathrm{C}$. The results showed that the addition of $\mathrm{NaF}$ to the reactive solution promotes the formation of a double layer of $\mathrm{MgF}_{2-x}(\mathrm{OH})_{x} / \mathrm{NaMgF}_{3}$. The presence of the $\mathrm{NaMgF}_{3}$ prevents the pitting corrosion attack of the coating/alloy system and leads a more uniform degradation mechanism. Particularly, the coating synthesized using $\mathrm{H}_{3} \mathrm{PO}_{4} 1.6 \% \mathrm{v}-\mathrm{NaF} 0.5 \mathrm{M}$ solution exhibited an excellent electrochemical behaviour, better that obtained employing HF solutions so that, it might be proposed as excellent candidate to replace the HF in the synthesis of biodegradable coatings.
\end{abstract}

Keywords: coating, alloy, magnesium, orthopedic implants, biodegradable, corrosion.

\title{
Resumo
}

$\mathrm{Na}$ atualidade, postularam-se ligas de magnésio como uma alternativa promissora na fabricação de implantes biodegradáveis por sua excelente degradabilidade, biocompatibilidade e propriedades mecânicas comparáveis às do osso. No entanto, sua elevada taxa de corrosão precisa a elaboração de revestimentos biodegradáveis, entre os quais destacam-se os sintetizados por conversão química em meio HF (até 48\%v). Porém, a utilização deste ácido gera grandes problemas em termos de segurança, pois é altamente tóxico. Isso leva à necessidade de encontrar formas alternativas para substituir o ácido ou limitar sua utilização em pequenas quantidades. Neste trabalho foram avaliados misturas HF (4\%v) $-\mathrm{NaF}$ e $\mathrm{H}_{3} \mathrm{PO}_{4}-\mathrm{NaF}$ como alternativas ao uso do $\mathrm{HF}$ na síntese de recobrimentos biodegradáveis na liga de magnésio Elektron 21. A caracterização microestrutural dos revestimentos foi realizada por microscopia eletrônica de varredura e difração de raios $\mathrm{X}$, enquanto a resistência à corrosão foi avaliada por testes electroquímicos e gravimétricos em solução de Hank a $27^{\circ} \mathrm{C}$. Os resultados mostraram que na presença de $\mathrm{NaF}$, os recobrimentos sintetizados são constituídos de uma bicamada $\mathrm{MgF}_{2-x}(\mathrm{OH})_{x} / \mathrm{NaMgF}_{3}$, onde a presença de NaMgF3 impede a corrosão localizada, causando que a degradação dos revestimentos seja uniforme e progressiva. Especialmente, os recobrimentos sintetizados sob a condição $\mathrm{H}_{3} \mathrm{PO}_{4} 1,6 \% \mathrm{v}-\mathrm{NaF}$ 0,5M mostraram excelente desempenho, superiores aos obtidos com a utilização do HF, portanto são recomendados como excelentes candidatos para a substituição imediata desse ácido.

Palabras-chave: revestimentos, liga, magnésio, implantes ortopédicos, biodegradáveis, corrosão.

\section{Introducción}

En la actualidad, gran número de personas poseen alguna parte de su cuerpo reparada o reemplazada artificialmente por piezas denominadas "implantes". Inicialmente, la investigación estaba enfocada a la fabricación de implantes ortopédicos temporales que proporcionaran soporte mecánico y ayudaran a mantener la funcionalidad del hueso durante el periodo de curación. Sin embargo, en el presente se busca la creación de piezas que, además, proporcionen el mínimo estrés fisiológico para el sistema óseo y eviten la realización de un segundo procedimiento quirúrgico para su extracción. Esto se puede lograr mediante el uso de materiales con características y propiedades mecánicas similares a las del tejido óseo, que se puedan degradar de forma controlada y cuyos productos derivados del proceso puedan ser absorbidos y/o excretados por el organismo de forma natural a medida que la parte afectada se recupera. 
En este sentido, el magnesio se posiciona como una excelente opción, ya que posee grandes ventajas con respecto a los materiales utilizados actualmente como son las aleaciones de $\mathrm{Ti}$, Co-Cr, y los aceros inoxidables austeníticos, materiales aceptados por el medio fisiológico a causa de la capa pasiva de óxido inerte formada en su superficie [1]. Sin embargo, por su diferencia de densidad y rigidez mecánica entre el hueso (10-20GPa) y el implante (100-200GPa), estos materiales protegen al hueso de recibir esfuerzos e impiden su adecuada regeneración, originando el fenómeno que se denomina "stress shielding" [2]. Además, por sus características químicas es necesaria una segunda cirugía para extraer el implante una vez el hueso ha sanado.

Entre las ventajas del magnesio se resalta su excelente biodegradabilidad en el medio fisiológico debido a que es esencial en muchas de las funciones biológicas de los seres humanos, entre ellas, como co-regulador del metabolismo energético, co-factor de varias enzimas, estabilizador de ADN y ARN y regulador de más de 350 proteínas, por lo que se requiere de un rango de consumo de 250 a $500 \mathrm{mg} /$ día [3-7]. Además, este elemento puede ser fácilmente eliminado a través de la orina, y posee propiedades osteoactivas necesarias para la regeneración ósea y la deposición de hidroxiapatita [3,4,8-9]. Por otro lado, sus características mecánicas, módulo elástico (20-40GPa) y su densidad próxima a la del hueso $\left(1,74\right.$ frente $1,80 \mathrm{~g} / \mathrm{cm}^{3}$ del hueso) reducen en gran medida el fenómeno de stress shielding y la pérdida de tejido óseo (osteopenia) [10].

Sin embargo, la principal desventaja del magnesio para su introducción en la fabricación de implantes ortopédicos es su elevada inestabilidad termodinámica. El magnesio es considerado uno de los metales más activos en la serie electroquímica con tendencia a transformarse espontáneamente hacia su estado oxidado y, por tanto, experimenta elevada velocidad de corrosión en medios fisiológicos, lo que impide mantener la integridad mecánica necesaria durante un periodo lo suficientemente largo para conseguir la adecuada reparación del hueso. Además, durante el proceso de corrosión se genera hidrógeno gaseoso que, en caso de acumulación, produce abultamientos cutáneos indeseados y necrosis del tejido circundante al ser aplicados in vivo [11-12]. En la búsqueda de elementos aleantes que permitan mejorar la estabilidad química del magnesio sin influir en su biocompatibilidad, se destaca el empleo de elementos de tierras raras (RE) y Zn que incrementan considerablemente la resistencia mecánica y a la corrosión-oxidación de aleaciones de magnesio, a la vez que exhiben alguna propiedad biocompatible. Tal es el caso de la aleación Elektron 21 (0,2-0,5\%Zn, 4,6-4,8\% $(\mathrm{Nd}+\mathrm{Gd})(\% \mathrm{p}))$, donde el gadolinio y neodimio mejoran la resistencia a la corrosión a la vez que presentan propiedades anticancerígenas, mientras que el zinc es añadido en bajas concentraciones para mejorar la resistencia mecánica y es esencial dentro del sistema inmunológico [2,13-16].

A pesar de ello, este tipo de aleaciones continúan presentado elevada actividad en fluidos fisiológicos. Es por ello que, para reducir la velocidad de corrosión a un valor tolerable, se requiere diseñar un recubrimiento superficial que actué como barrera protectora y permita controlar la cinética de degradación de la aleación como condición indispensable antes que pueda emplearse el magnesio como material en la fabricación de implantes ortopédicos temporales biodegradables. Sin embargo, el reto al que se enfrentan los investigadores es diseñar un recubrimiento que no solamente brinde protección, sino que además posea carácter biodegradable, de tal manera que se disuelva gradualmente a medida que el implante cumpla su función.

Los recubrimientos biodegradables que han sobresalido hasta la fecha se han logrado en su mayoría por conversión química. Estos cuentan con el atributo de ser de bajo costo, fácil aplicación, tener una gran adherencia, homogeneidad y presentar una excelente resistencia a la corrosión [17-21]. Los recubrimientos por conversión se forman por reacción entre el material base y el medio utilizado para este fin, de tal forma que, dependiendo del medio, se formarán diferentes tipos de recubrimientos [12]. En este sentido, se ha encontrado que el uso de ácidos orgánicos como el ácido fítico o ácido esteárico o sales como fosfatos, permanganatos, estanatos y fluoruros, crea capas protectoras biodegradables [19,22-25]. Dentro de este tipo de recubrimientos son de especial interés los recubrimientos de fluoruro de magnesio $\left(\mathrm{MgF}_{2}\right)$ debido a su carácter biocompatible y su potencial carácter biodegradable. Estudios recientes han demostrado que los recubrimientos de $\mathrm{MgF}_{2}$ desarrollados sobre diferentes tipos de piezas presentan como resultado un excelente acabado superficial y un proceso de biodegradación bastante prometedor, que resulta de la acción competitiva entre los 
iones fluoruro $\left(\mathrm{F}^{-}\right)$y los iones presentes en el medio fisiológico; de esta forma, a medida que progresa el proceso de corrosión, la película de $\mathrm{MgF}_{2}$ se va disolviendo progresivamente hasta desaparecer por completo, momento en el que se comienza a observar la respuesta del material base [26-27]. Finalmente, otra de las características que hacen idóneos a dichos recubrimientos es el propio anión fluoruro, debido a que éste aumenta la proliferación de células osteoblásticas, incrementa la deposición de nuevos minerales en el hueso y es esencial para el crecimiento normal del sistema óseo $[20,26]$.

Para la síntesis de estos recubrimientos, el precursor más comúnmente utilizado es el ácido fluorhídrico que, por lo general, es trabajado en altas concentraciones que van desde el 4 al $48 \% \mathrm{v}$, condiciones bajo las que se han obtenido aumentos significativos de la resistencia a la corrosión, de más de un orden de magnitud, y un ataque mucho más uniforme y suave con respecto a la aleación de magnesio sin tratamiento [26,28-31]. Sin embargo, el manejo de altas concentraciones de HF conlleva a grandes riesgos para la salud de los operarios al momento de manipular esta sustancia durante la producción de los recubrimientos, pues es sabido que el HF además de ser muy corrosivo, es altamente tóxico al ser inhalado o ingerido, causando daño a los tejidos, particularmente a las mucosas de los ojos, la boca y al tracto respiratorio. Es por ello que existe la necesidad de hallar vías alternativas que permitan sustituir dicho ácido, o en su defecto limitar su uso a cantidades reducidas.

Actualmente, se están buscando rutas alternativas para el reemplazo inmediato de HF por otro tipo de sustancias menos peligrosas. Entre una de las alternativas que se plantea es el empleo de sales de fluoruro como $\mathrm{NaF}$ y KF de menor toxicidad y costo, junto con un medio ácido precursor que permita controlar el pH del medio de síntesis [22]. Con base en lo anterior, el objetivo del presente trabajo fue evaluar dos vías alternativas de síntesis de recubrimientos $\mathrm{MgF}_{2}$ sobre la aleación Elektron 21, que permitan sustituir bien sea total o parcialmente, el empleo de HF. En este sentido, se propusieron dos rutas alternativas de síntesis: i) mezclas HF-NaF (sustitución parcial de HF) y ii) mezclas $\mathrm{H}_{3} \mathrm{PO}_{4}-\mathrm{NaF}$ (sustitución completa de $\mathrm{HF}$ ) que fueron comparadas con dos condiciones de HF (4 y $10 \% \mathrm{v})$ y se establecieron con base en los excelentes resultados obtenidos en anteriores investigaciones sobre la misma aleación [32].

\section{Procedimiento experimental}

\section{Material objeto de estudio}

El material utilizado en la presente investigación fue la aleación de magnesio denominada comercialmente Elektron 21 [33], cuya composición química se presenta en la Tabla 1.

Tabla 1.Composición nominal de la aleación Elektron 21 (\%p)

\begin{tabular}{cccccc}
\hline Designación & $\boldsymbol{N d}$ & $\mathbf{G d}$ & $\mathbf{Z n}$ & $\mathbf{Z r}$ & $\mathbf{M g}$ \\
\hline Elektron 21 & $2,6-3,1$ & $1,0-1,7$ & $0,2-0,5$ & Saturado & Balance \\
\hline
\end{tabular}

\section{Caracterización microestructural del material base}

Las muestras se prepararon superficialmente mediante desbaste gradual con papel de carburo de silicio, $\mathrm{SiC}$, hasta granulometría 600 , seguido de un pulido con pasta de diamante de 3 y $1 \mu \mathrm{m}$ hasta obtener una superficie especular. Posteriormente, con objeto de revelar su microestructura, las muestras fueron atacadas ligeramente con solución de ácido pícrico (10ml ácido acético, $6 \mathrm{~g}$ ácido pícrico, 100ml etanol) según la norma ASTM E-407 "Standard Practice for Microetching Metals and Alloys" [34]. La caracterización microestructural del material base se llevó a cabo empleando un microscopio electrónico de barrido
Quanta FEG 650, equipado con detectores para el análisis de energías dispersivas de rayos $X$ (EDX) y para la obtención de imágenes por electrones retrodispersados (BSE).

\section{Síntesis del recubrimiento por conversión química}

Previo a la síntesis del recubrimiento, las muestras fueron preparadas superficialmente con papel de SiC hasta granulometría 600 , limpiadas en baño ultrasónico con etanol al $96 \%$ v durante 2 minutos y finalmente secadas con aire caliente. La síntesis del recubrimiento por conversión química se llevó a cabo mediante la inmersión de las muestras en diferentes soluciones acuosas conteniendo ión 
fluoruro. Se estableció como variables de estudio el tipo y concentración tanto de ácido precursor como de fuente de ion fluoruro empleados, siendo diferente en cada ruta de síntesis como se muestra en la Tabla 2. Como recubrimientos base de referencia se seleccionaron las condiciones
HF al 4 y $10 \%$ v. La temperatura y el tiempo de inmersión en la solución de síntesis se fijaron en $25^{\circ} \mathrm{C}$ (temperatura ambiente) y 24 horas, respectivamente. Después de la inmersión, las muestras se lavaron con agua destilada y se secaron en una estufa a $120^{\circ} \mathrm{C}$ durante 30 minutos.

Tabla 2.Concentraciones de reactivos empleados en cada ruta de síntesis propuesta.

\begin{tabular}{ccccc}
\hline Ruta de síntesis & Ácido precursor & Concentración & Fuente de fluoruro alternativa & Concentración \\
\hline $\mathbf{H F}$ & $\mathrm{HF}$ & $4,0 \% \mathrm{v}$ & - & - \\
& & $10,0 \% \mathrm{v}$ & $\mathrm{NaF}$ & \\
$\mathrm{HF}+\mathrm{NaF}$ & $\mathrm{HF}$ & $4,0 \% \mathrm{v}$ & & $0,1 \mathrm{M}^{(*)}$ \\
& & & & $0,3 \mathrm{M}$ \\
& & & $\mathrm{NaF}$ & $0,5 \mathrm{M}$ \\
$\mathbf{H}_{3} \mathrm{PO}_{4}+\mathrm{NaF}$ & $\mathrm{H}_{3} \mathrm{PO}_{4}$ & $1,1 \% \mathrm{v}$ & & $0,1 \mathrm{M}^{(*)}$ \\
& & $1,6 \% \mathrm{v}$ & $0,3 \mathrm{M}$ \\
& & & & $0,5 \mathrm{M}$ \\
\hline
\end{tabular}

$\left.{ }^{*}\right)$ Los recubrimientos preparados con $\mathrm{NaF} 0,1 \mathrm{M}$ mostraron un severo ataque corrosivo superficial por lo que dichas condiciones de síntesis se descartaron para estudios posteriores.

\section{Caracterización micro-estructural del recubrimiento \\ La caracterización microestructural de los recubrimientos se efectuó empleando el mismo microscopio electrónico empleado en la caracterización del material base. Complementariamente, con objeto de identificar el tipo de fases formadas durante la síntesis, así como su carácter cristalino, se empleó difracción de rayos $X(D R X)$ de incidencia rasante $\left(2^{\circ}\right)$ mediante el difractómetro BRUKER modelo D8DISCOVER con radiación de CuKa1 y filtro de níquel.}

\section{Evaluación de la resistencia a la corrosión}

Para evaluar la protección frente al fenómeno de corrosión ofrecida por los recubrimientos se realizaron ensayos gravimétricos y ensayos electroquímicos de polarización anódica potenciodinámica. Los ensayos fueron realizados a temperatura de $37 \pm 1^{\circ} \mathrm{C}$ (temperatura corporal) en solución Hank (solución fisiológica simulada), de pH 7,79 y la siguiente composición: $8 \mathrm{~g} / \mathrm{l} \mathrm{NaCl}, 0,19 \mathrm{~g} / \mathrm{l}$ $\mathrm{CaCl}_{2}, 0,4 \mathrm{~g} / \mathrm{KCl}, 0,35 \mathrm{~g} / \mathrm{l} \mathrm{NaHCO}{ }_{3}, 1 \mathrm{~g} / \mathrm{l}$ glucosa, $0,19 \mathrm{~g} / \mathrm{l} \mathrm{MgCl} \cdot 6 \mathrm{H}_{2} \mathrm{O}, 0,06 \mathrm{~g} / \mathrm{l} \mathrm{Na}{ }_{2} \mathrm{HPO}_{4} 2 \mathrm{H}_{2} \mathrm{O}, 0,06 \mathrm{~g} / \mathrm{l}$ $\mathrm{KH}_{2} \mathrm{PO}_{4}, 0,06 \mathrm{~g} / \mathrm{l} \mathrm{MgSO}{ }_{4} \cdot 7 \mathrm{H}_{2} \mathrm{O}[35]$.

Ensayos gravimétricos. Se realizaron ensayos gravimétricos de corrosión de la aleación base con y sin recubrimiento bajo las especificaciones de las normas ASTMG1-90 y G31-72 [36,37]. Las muestras, una vez determinadas la masa y el área de exposición, fueron sumergidas en solución Hank por un tiempo total de 30 días, durante los cuales fueron extraídas a diferentes tiempos de inmersión, para ser posteriormente lavadas con agua destilada, secadas en estufa a $120^{\circ} \mathrm{C}$ durante 30 minutos $\mathrm{y}$, finalmente, pesadas con objeto de evaluar la variación de masa a lo largo del proceso de corrosión. Así mismo, con el fin de determinar la morfología de los productos de corrosión y el mecanismo de corrosión, muestras con diferentes tiempos de inmersión en el medio corrosivo fueron analizadas mediante microscopía electrónica de barrido.

Polarización anódica. Los ensayos de polarización anódica potenciodinámica se realizaron con el potenciostato GAMRY 600. Se diseñó una celda de tres electrodos donde se empleó un electrodo de plata-cloruro de plata $(\mathrm{Ag} / \mathrm{AgCl})$ como electrodo de referencia, platino (Pt) como electrodo auxiliar y la muestra a evaluar como electrodo de trabajo. El área de exposición de las muestras fue de $1 \mathrm{~cm}^{2}$. Cada experimento tuvo un tiempo de estabilización del potencial a circuito abierto (OCP) de 30 minutos. Una vez alcanzado dicho tiempo, se procedió a realizar el ensayo de polarización anódica en el que se llevó a cabo un barrido anódico de potencial desde -100 hasta $2000 \mathrm{mV}$ (con respecto al potencial 
de circuito abierto (OCP)), con una velocidad de barrido de $0,3 \mathrm{mV} / \mathrm{s}$ y una densidad de corriente límite de $1 \mathrm{~mA} / \mathrm{cm}^{2}$.

\section{Resultados y Discusión}

\section{Caracterización microestructural de la aleación Elektron 21}

En la Figura 1 se presenta la micrografía electrónica de la aleación de magnesio Elektron 21 donde se observa que presenta una microestructura típica de moldeo, constituida por granos equiaxiales y homogéneos de $\alpha-M g$, con presencia de las fases intermetálicas $\mathrm{Mg}_{12}\left(\mathrm{Nd}_{\mathrm{x}}, \mathrm{Gd}_{1-\mathrm{x}}\right)$ precipitada en los límites de grano, y $\mathrm{Zr}-\mathrm{Zn}$ en el interior del grano con morfología acicular [38]. Por otro lado, también es posible observar en el centro de los granos algunas partículas cuasi esféricas principalmente compuestas de $\mathrm{Zr}$ y de tamaño cercano a $1 \mu \mathrm{m}$.

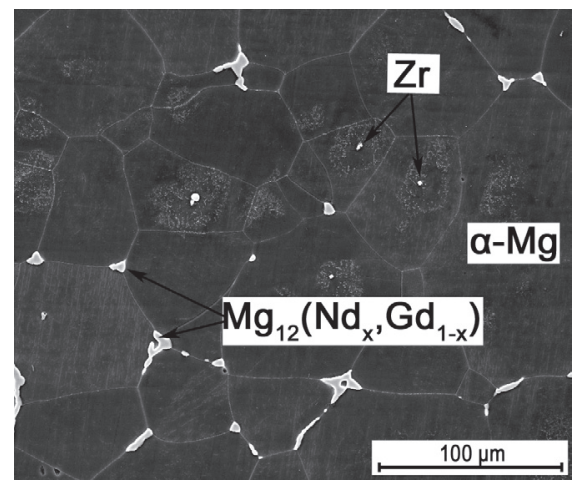

Figura 1. Micrografía electrónica de barrido de la aleación Elektron 21.

\section{Caracterización del recubrimiento}

Las micrografías electrónicas mostradas en la Figura 2 muestran los recubrimientos sintetizados empleando HF al 4 y $10 \%$ v tanto superficiales como en su sección transversal. Los resultados revelaron que para ambas condiciones de síntesis los recubrimientos presentan una apariencia continua y homogénea, con un espesor de alrededor de $2 \mu \mathrm{m}$. Así mismo, se observa que las fases intermetálicas $\mathrm{Mg}_{12}\left(\mathrm{Nd}_{\mathrm{x}}, \mathrm{Gd}_{1-\mathrm{x}}\right)$ permanecen sin disolver formando parte de la estructura del recubrimiento. Por otro lado, un análisis EDX realizado al recubrimiento muestra la presencia de $\mathrm{Mg}, \mathrm{F}$ y $\mathrm{O}$, indicando la presencia de una composición $\mathrm{MgF}_{2-\mathrm{x}}(\mathrm{OH})_{\mathrm{x}}$ (hidroxifluoruro), donde los aniones hidroxilo y fluoruro se incorporan a la red del recubrimiento durante su formación, hecho ya reportado por otros autores [26,30,31].

Con relación a los recubrimientos sintetizados en soluciones $\mathrm{HF}(4 \% \mathrm{v})$ con adición de $\mathrm{NaF}$, se debe mencionar inicialmente que los recubrimientos sintetizados con $0,1 \mathrm{M}$ de $\mathrm{NaF}$ (tanto en HF como $\mathrm{H}_{3} \mathrm{PO}_{4}$ ) mostraron corrosión severa superficial por lo que fueron descartados para estudios posteriores. Probablemente, la concentración de $\mathrm{NaF}$ es insuficiente para contrarrestar el efecto corrosivo del ácido precursor, y por tanto, no beneficia la formación de la película protectora. Sin embargo, para concentraciones superiores, se observa una aparente continuidad y homogeneidad estructural. No obstante, un análisis más detallado detecta la formación de precipitados con geometría cristalina sobre la superficie, siendo su presencia más abundante a medida que se incrementa el contenido de $\mathrm{NaF}$ en la solución (Figuras 3a y 3b). Un estudio de la sección transversal muestra la formación de dos capas, una interna continua de menor espesor (500 nm aprox.) que las observadas para soluciones de HF, por encima de la cual tiene lugar la formación de los cristales anteriormente mencionados (Figura 3c). El análisis EDX en dicha región permite identificar que la película interna corresponde al hidroxifluoruro de magnesio $\left(\mathrm{MgF}_{2-x}(\mathrm{OH})_{\mathrm{x}}\right)$, con una estequiometria que por la variación observada en la concentración del flúor y el oxígeno, se deduce que no es fija; mientras que los cristales presentan en su composición $\mathrm{Na}, \mathrm{Mg}$ y F, posiblemente $\mathrm{NaMgF}_{3}$ (neighborita) (Figuras 3d y $3 e$ ). Resultados similares fueron publicados en un estudio reciente basado en el empleo de soluciones de KF [10] donde se indicó la formación de una bicapa donde la película interna era principalmente amorfa constituida por una mezcla de $\mathrm{Mg}(\mathrm{OH})_{2}$ y $\mathrm{MgF}_{2}$, mientras que la película externa estaba constituida por $\mathrm{KMgF}_{3}$ cristalino. Los autores plantean en dicho estudio que la fase $\mathrm{KMgF}_{3}$ es formada por interacción del $\mathrm{MgF}_{2}$ precipitado y el KF presente en la solución de síntesis. 

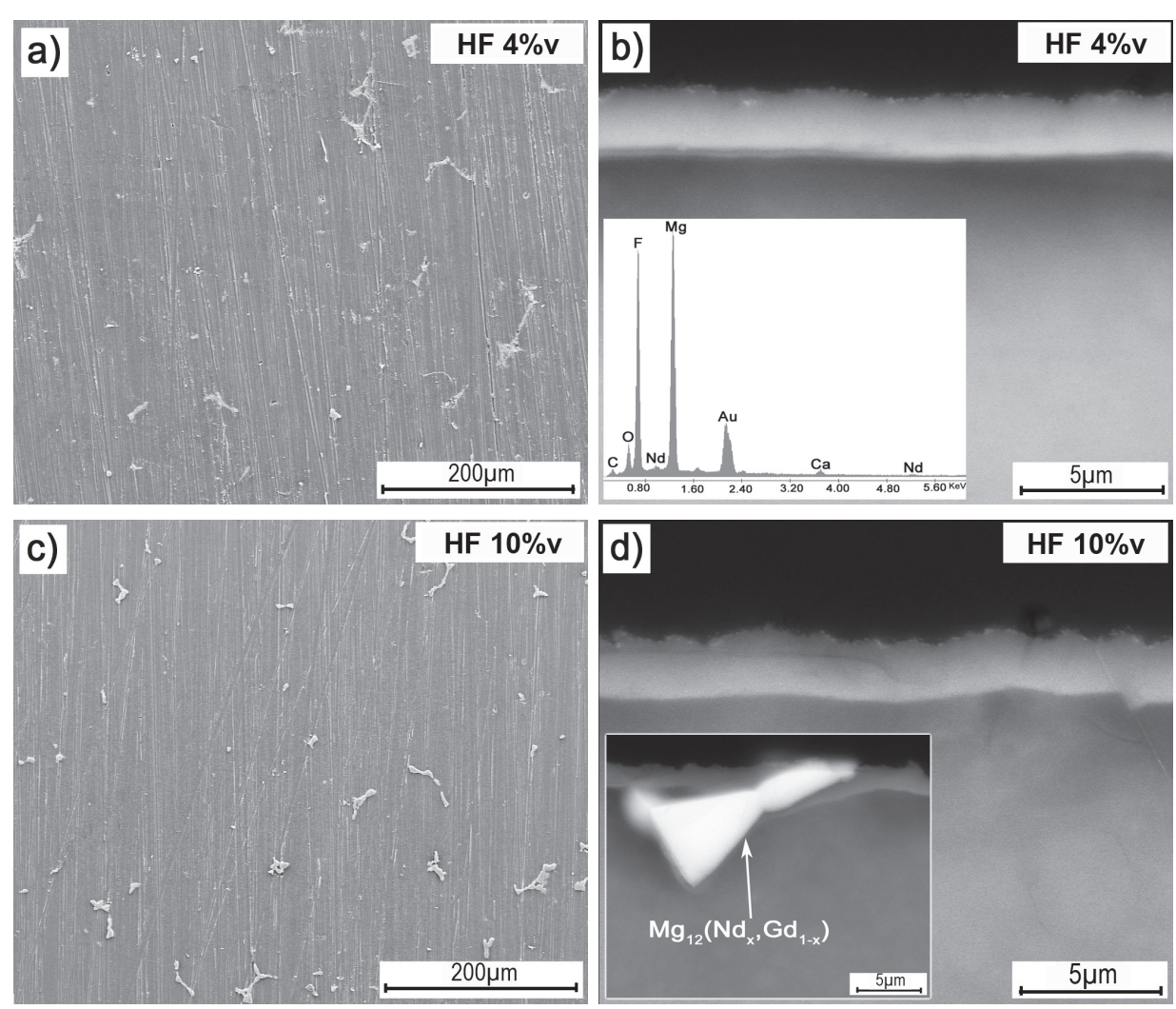

Figura 2. Micrografías electrónicas superficiales y transversales de las muestras recubiertas por conversión química en: a) y b) $4 \%$ v HF; c) y d) $10 \%$ v HF.
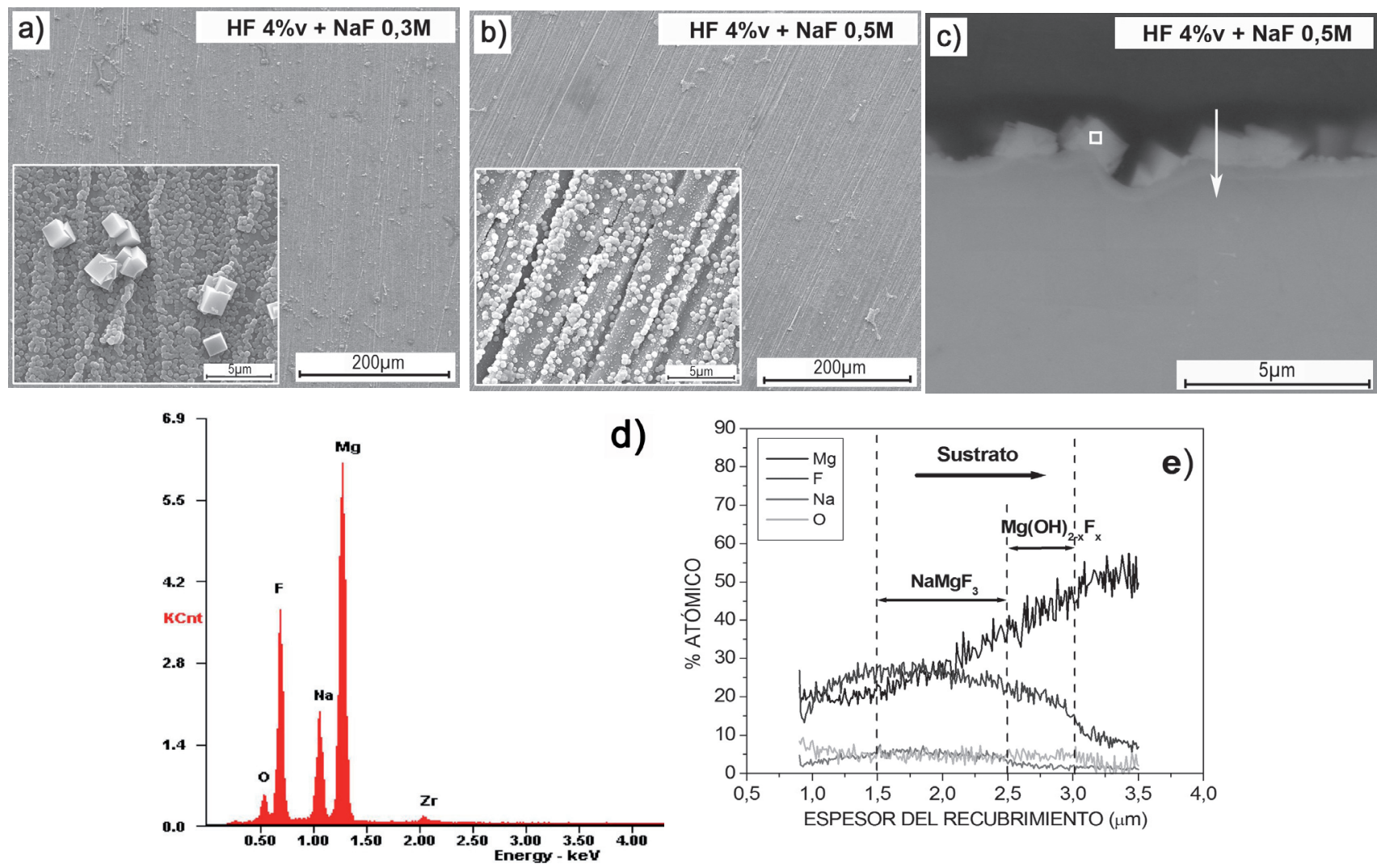

Figura 3. Micrografías electrónicas superficiales de las muestras recubiertas por inmersión en 4\%v HF con adición de NaF: a) 0,3M y b) 0,5M; c), d) y e) Análisis MEB-EDX de la sección transversal del recubrimiento sintetizado bajo la condición $4 \%$ v HF- 0,5M NaF. 
Finalmente, se muestran las micrografías electrónicas superficiales de los recubrimientos sintetizados empleando como precursores $\mathrm{H}_{3} \mathrm{PO}_{4}$ y $\mathrm{NaF}$ (Figura 4). Se puede apreciar que a medida que se incrementa la concentración de $\mathrm{H}_{3} \mathrm{PO}_{4}$ y $\mathrm{NaF}$ en el medio, la formación de cristales superficiales es cada vez más elevada, de manera que, para la condición $\mathrm{H}_{3} \mathrm{PO}_{4} \quad 1,6 \% \mathrm{v}$ y $0,5 \mathrm{M} \mathrm{NaF}$, la película externa es prácticamente continua sobre toda la superficie de la aleación. Un estudio en mayor detalle de la superficie permite, además, observar la disolución de la fase
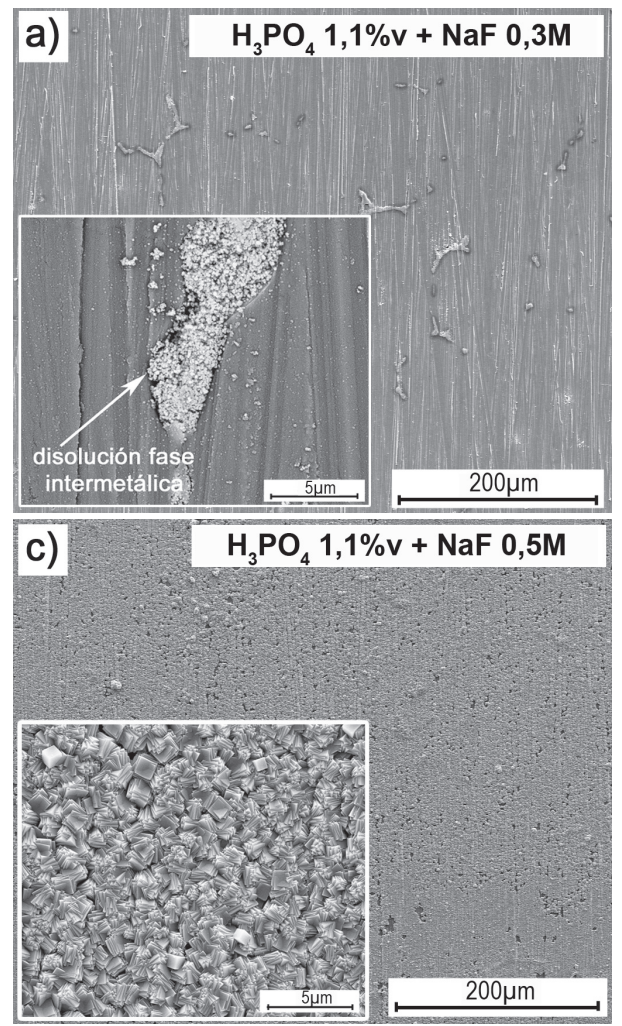

intermetálica, que posiblemente no es estable en medio $\mathrm{H}_{3} \mathrm{PO}_{4}$ (Figura 4a), y por otro lado, que los recubrimientos están constituidos por dos capas: i) una primera capa uniforme que crece directamente sobre el sustrato, y ii) una segunda capa, formada por pequeños cristales que nuclean en forma esférica, y crecen sobre la primera capa hasta alcanzar la morfología cúbica (Figura 4b). La homogeneidad y compacidad de esta segunda capa, aumentan directamente con el incremento en las concentraciones de ácido y de $\mathrm{NaF}$ (Figuras $4 c$ y $4 d$ ).
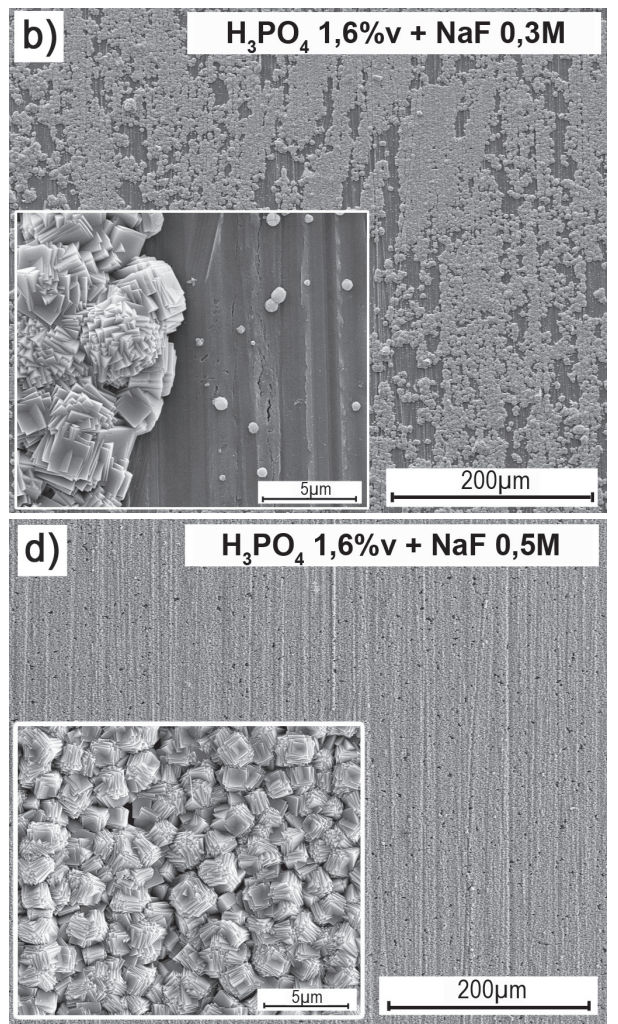

Figura 4. Micrografías electrónicas superficiales de las muestras recubiertas por conversión química bajo diferentes condiciones de síntesis en medios $\mathrm{H}_{3} \mathrm{PO}_{4}-\mathrm{NaF}$.

Para determinar la naturaleza composicional de las capas formadas se realizaron análisis de DRX de incidencia rasante sobre la aleación base y las muestras recubiertas tanto con HF como con $\mathrm{H}_{3} \mathrm{PO}_{4}$. Los resultados se presentan en la Figura 5. De este modo, la muestra tratada con HF (Figura 5a), presenta máximos de difracción representativos del $\mathrm{Mg}$ y algunos de menor intensidad a $27,38,41,44$ y $54^{\circ}$ correspondiente a $\mathrm{MgF}_{2-x}(\mathrm{OH})_{x}$ (que presenta similar estructura cristalina que la sellaíta, $\mathrm{MgF}_{2}$ ), cuyos picos son poco definidos debido a la estequiometria variable de este compuesto en el recubrimiento, como se determinó con anterioridad (Figura 3e). Por otro lado, los recubrimientos sintetizados a partir de HF con adición de $\mathrm{NaF}$ muestran en menor medida la presencia de $\mathrm{MgF}_{2-x}(\mathrm{OH})_{\mathrm{x}}$, mientras que los picos de difracción correspondientes a $\mathrm{NaMgF}_{3}$ aún no se alcanzan a observar debido a la baja concentración de este compuesto. En cuanto a los recubrimientos sintetizados en solución $\mathrm{H}_{3} \mathrm{PO}_{4}$, la presencia de $\mathrm{NaMgF}_{3}$, con máximos de difracción localizados a 39,48 y $59^{\circ}$, se hace evidente a medida que se incrementa la concentración tanto de ácido fosfórico como de $\mathrm{NaF}$, lo que confirma los resultados obtenidos por MEB (Figura $5 b$ ). 

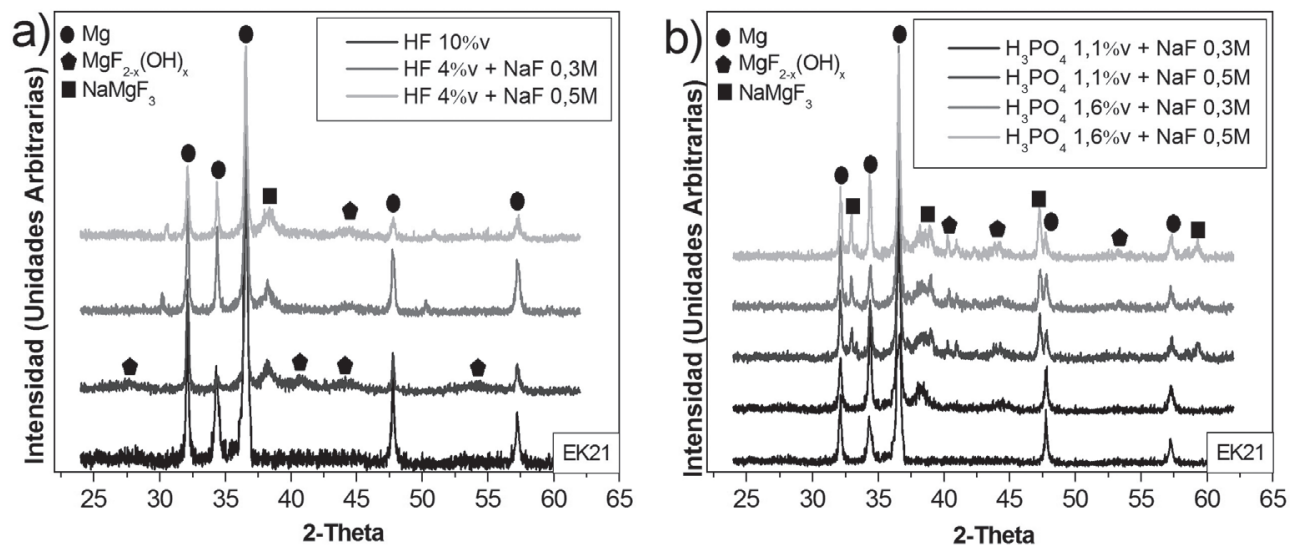

Figura 5. Difractogramas de rayos $\mathrm{X}$ de la aleación sin tratar y recubierta bajo diferentes condiciones de síntesis: a) medio $\mathrm{HF} \mathrm{y} \mathrm{b)} \mathrm{medio} \mathrm{H}_{3} \mathrm{PO}_{4}$.

A partir de los anteriores resultados se postula un mecanismo de formación de los recubrimientos sintetizados en presencia de $\mathrm{NaF}$ mediante el siguiente esquema de reacciones:

$$
\begin{gathered}
\mathrm{Mg} \rightarrow \mathrm{Mg}^{2+}+2 \mathrm{e}^{-} \\
2 \mathrm{H}_{2} \mathrm{O}+2 \mathrm{e}^{-} \rightarrow \mathrm{H}_{2} \uparrow+2 \mathrm{OH}^{-} \\
\mathrm{Mg}^{2+}+\mathrm{OH}^{-} / \mathrm{F}^{-} \rightarrow \mathrm{MgF}_{2-\mathrm{x}}(\mathrm{OH})_{\mathrm{x}} \downarrow \\
\mathrm{MgF}_{2-\mathrm{x}}(\mathrm{OH})_{\mathrm{x}}+\mathrm{NaF} \rightarrow \mathrm{NaMgF}_{3}+\mathrm{OH}^{-}
\end{gathered}
$$

Donde las Ecuaciones 1 y 2 corresponden a las reacciones anódica y catódica respectivamente, propias del proceso de corrosión experimentado por la aleación de magnesio en contacto con cualquiera de los dos ácidos estudiados. Por otro lado, la Ecuación 3 representa la formación de la película mixta de hidroxifluoruro de magnesio que se deposita sobre la superficie de la aleación en presencia de aniones hidroxilo y fluoruro disponibles en solución. Finalmente, en contacto con el $\mathrm{NaF}$, dicha película es consumida parcialmente y se transforma para dar lugar a la película de neighborita, $\mathrm{NaMgF}_{3}$, tal como lo describe la Ecuación 4.

\section{Evaluación de la resistencia a la corrosión Ensayo de polarización anódica} potenciodinámica. En la Figura 6 se muestran las curvas obtenidas por medio de los ensayos de polarización anódica potenciodinámica de la aleación de estudio Elektron 21 sin recubrimiento y recubierta bajo diferentes condiciones de síntesis. $A$ través de las curvas de polarización se determinaron parámetros electroquímicos de interés, que se encuentran contenidos en la Tabla 3.
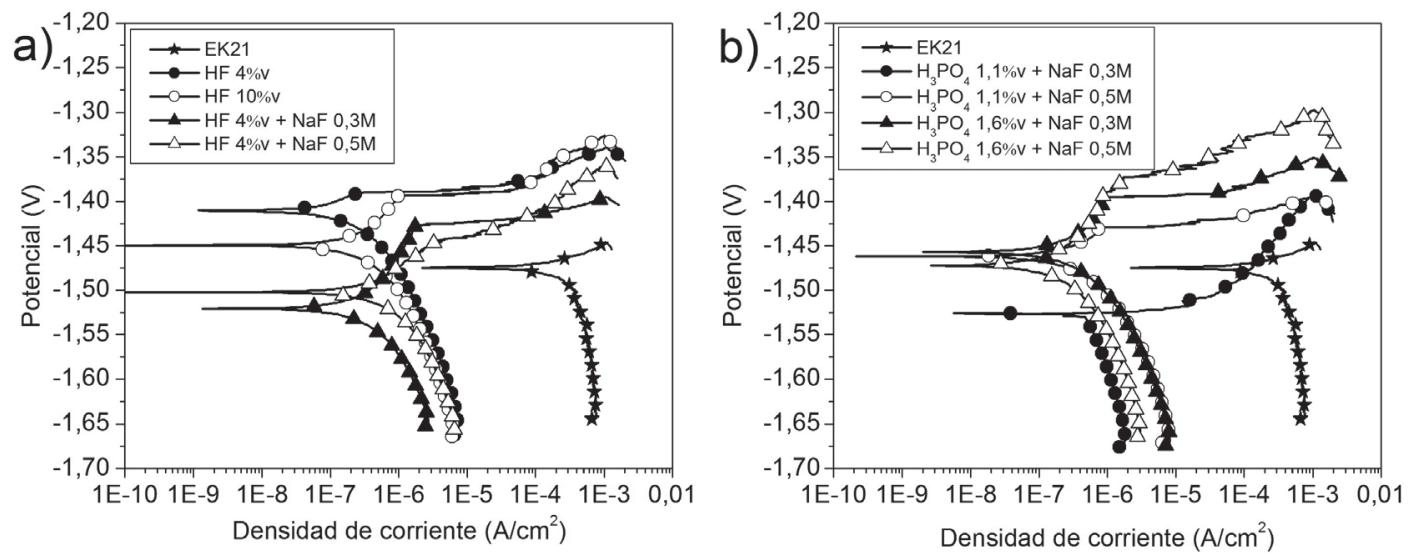

Figura 6. Curvas de polarización anódica de la aleación Elektron 21 sin recubrimiento y recubierta bajo diferentes condiciones de síntesis: a) medio $\mathrm{HF}$ y b) medio $\mathrm{H}_{3} \mathrm{PO}_{4}$. 
rev.ion. 2015;28(2):7-21. Bucaramanga (Colombia).

Tabla 3. Parámetros electroquímicos obtenidos de las curvas de polarización anódica presentadas en la Figura 6.

\begin{tabular}{ccccc}
\hline Relaciones & $\mathbf{i}_{\text {corr }}\left(\mathbf{A} / \mathbf{c m}^{2}\right)$ & $\mathbf{E}_{\text {corr }}(\mathbf{V})$ & $\mathbf{E}_{\text {pic }}(\mathbf{V})$ & $\mathbf{E}_{\text {pic }}-\mathbf{E}_{\text {corr }}(\mathbf{V})$ \\
\hline Material Base & $1,36 \times 10^{-04}$ & $-1,47$ & --- & --- \\
HF 4\%v & $4,26 \times 10^{-08}$ & $-1,41$ & $-1,39$ & 0,02 \\
HF 10\%v & $8,99 \times 10^{-08}$ & $-1,45$ & $-1,39$ & 0,06 \\
$\mathrm{HF} 4 \% \mathrm{v}+\mathrm{NaF0,3M}$ & $1,92 \times 10^{-07}$ & $-1,52$ & $-1,43$ & 0,09 \\
$\mathrm{HF} 4 \% \mathrm{~V}+\mathrm{NaF0,5M}$ & $2,60 \times 10^{-07}$ & $-1,50$ & $-1,44$ & 0,06 \\
$\mathrm{H}_{3} \mathrm{PO}_{4} 1,1 \% \mathrm{v}+\mathrm{NaF} 0,3 \mathrm{M}$ & $4,74 \times 10^{-07}$ & $-1,53$ & $-1,52$ & 0,01 \\
$\mathrm{H}_{3} \mathrm{PO}_{4} 1,1 \% \mathrm{~N}+\mathrm{NaF} 0,5 \mathrm{M}$ & $1,67 \times 10^{-07}$ & $-1,46$ & $-1,43$ & 0,03 \\
$\mathrm{H}_{3} \mathrm{PO}_{4} 1,6 \% \mathrm{~V}+\mathrm{NaF} 0,3 \mathrm{M}$ & $1,36 \times 10^{-07}$ & $-1,46$ & $-1,39$ & 0,07 \\
$\mathrm{H}_{3} \mathrm{PO}_{4} 1,6 \% \mathrm{~V}+\mathrm{NaF} 0,5 \mathrm{M}$ & $9,40 \times 10^{-08}$ & $-1,47$ & $-1,39$ & 0,08 \\
\hline
\end{tabular}

La aleación base sin recubrimiento no presenta potencial de picadura $\left(E_{\text {pic }}\right)$, lo que indica que el material experimenta corrosión por picadura libremente en la solución Hank. Por el contrario, todas las muestras recubiertas presentan potencial de picadura y zona de pasivación $\left(\mathrm{E}_{\mathrm{pic}}-\mathrm{E}_{\text {corr }}\right)$, así como una reducción de la densidad de corriente de corrosión ( $\mathrm{i}_{\text {corr }}$ ) de entre 3 y 4 órdenes de magnitud, situándose en valores alrededor de $1 \times 10^{-07} \mathrm{~A} / \mathrm{cm}^{2}$, para todas las condiciones evaluadas. Ambos hechos son indicativos de que los recubrimientos ofrecen un excelente carácter protector al sustrato metálico. Por otro lado, se destaca el hecho de que los recubrimientos sintetizados en presencia $\mathrm{H}_{3} \mathrm{PO}_{4} 1,6 \%$ v y las diferentes concentraciones de $\mathrm{NaF}$, mejoran significativamente las características pasivas de la aleación Elektron 21, incluso por encima de los recubrimientos sintetizados con HF $10 \% \mathrm{v}$, lo que probablemente esté relacionado con la presencia de neighborita en su composición. A partir de los anteriores resultados se puede concluir, que los recubrimientos sintetizados con la alternativa propuesta $\left(\mathrm{NaF}-\mathrm{H}_{3} \mathrm{PO}_{4} 1,6 \% \mathrm{v}\right)$ presentan características protectoras de calidad similar o superior a los obtenidos con el empleo de HF, por lo que son excelentes candidatos en términos electroquímicos para la sustitución de este ácido, evitando los riesgos asociados a su manipulación.

Ensayos gravimétricos. La Figura 7 muestra los resultados de los ensayos gravimétricos realizados, donde se observa que, en general, todas las muestras presentan ganancia en masa, asociada a la formación de $\mathrm{Mg}(\mathrm{OH})_{2}$ como principal producto de corrosión, siendo su formación más pronunciada para la muestra recubierta con $\mathrm{H}_{3} \mathrm{PO}_{4}$ y NaF 0,3M. Por otro lado, para las concentraciones de $\mathrm{H}_{3} \mathrm{PO}_{4}$ y $\mathrm{NaF} 0,5 \mathrm{M}$ se presentan los mejores resultados, exhibiendo una menor ganancia en masa respecto a la aleación base sin recubrir y a las muestras tratadas con $\mathrm{HF}$ al 4 y $10 \%$, hecho que probablemente esté atribuido a que estos recubrimientos, según el estudio de caracterización, presentaron una mayor formación de neighborita superficial cuyas características morfológicas podrían haber incrementado su carácter protector.

La Figura 8 presenta las macrografías de varias muestras ensayadas gravimétricamente a diferentes tiempos de inmersión, donde se observa físicamente el avance del ataque y la formación de productos de corrosión. En el caso de las muestras recubiertas con HF $10 \%$ v y $\mathrm{HF}$ con adición de $\mathrm{NaF}$, se observa la formación de cúmulos blancos de $\mathrm{Mg}(\mathrm{OH})_{2}$ que se hacen más evidentes a medida que evoluciona el proceso de degradación y que posiblemente esté relacionada con un mecanismo de corrosión localizada por picadura del recubrimiento en el medio de ensayo. Así mismo, se observan zonas con mayor grado de degradación que alcanza a disolver incluso el sustrato metálico (Figuras $8 a$ y $8 b$ ). Por otra parte, la muestra tratada con bajas concentraciones de $\mathrm{H}_{3} \mathrm{PO}_{4}$ y $\mathrm{NaF} 0,3 \mathrm{M}$ (Figura 8c) muestra signos evidentes de ataque localizado desde el primer día, con presencia de cúmulos blancos correspondientes a núcleos de corrosión que con el paso del tiempo crecen generando una película que cubre por completo la superficie del material. Por el contrario, los recubrimientos obtenidos con $\mathrm{H}_{3} \mathrm{PO}_{4}-\mathrm{NaF}$ con las mayores concentraciones de ambos compuestos, presentan una degradación más uniforme y progresiva, sin evidencia de corrosión localizada, cuyo recubrimiento se disuelve paulatinamente dejando la aleación base expuesta para su posterior disolución (Figura 8d). 

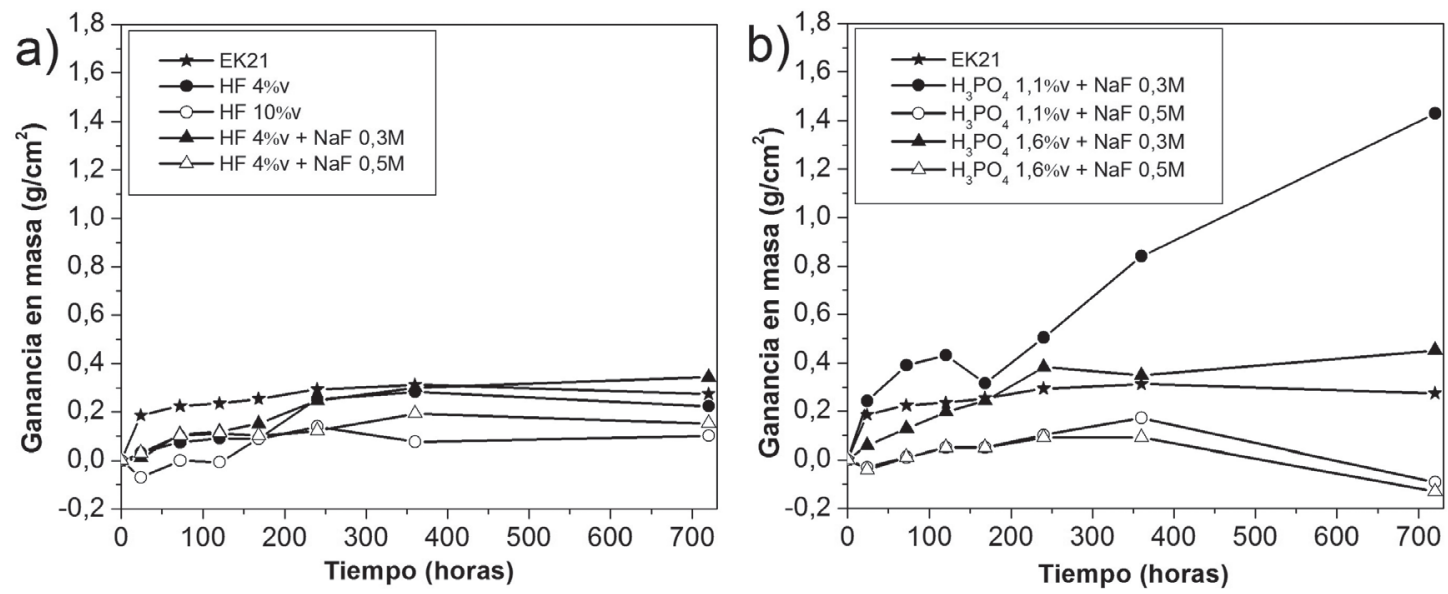

Figura 7. Evolución de la ganancia en masa por unidad de área durante inmersión en solución Hank de la aleación Elektron 21 sin recubrimiento y recubierta bajo diferentes condiciones de síntesis.

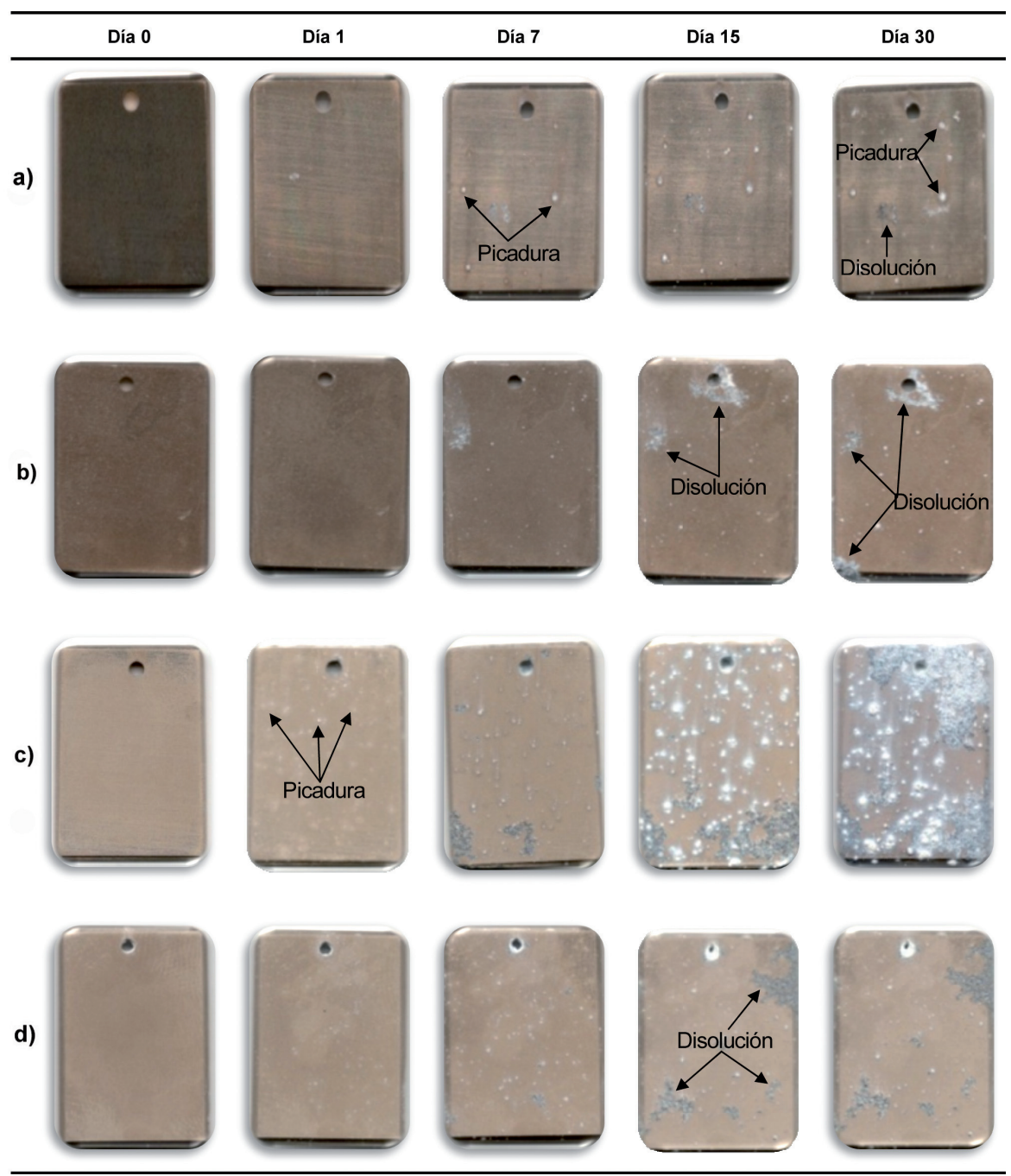

Figura 8. Macrografías superficiales de los recubrimientos sintetizados después de inmersión en solución Hank durante diferentes tiempos: a) $\mathrm{HF} 10 \% \mathrm{v}$; b) $\mathrm{HF} 4 \% \mathrm{v}+\mathrm{NaF} 0,5 \mathrm{M}$; c) $\mathrm{H}_{3} \mathrm{PO}_{4} 1,1 \% \mathrm{v}+\mathrm{NaF} 0,3 \mathrm{M}$; d) $\mathrm{H}_{3} \mathrm{PO}_{4} 1,6 \% v+$ $\mathrm{NaF} 0,5 \mathrm{M}$. 
Finalmente, con el fin de determinar el inicio de ataque de las muestras y la morfología de los productos de corrosión, se realizó un análisis mediante MEB-EDX de las muestras expuestas a un día de inmersión en solución Hank. Se observa que las muestras tratadas con HF (Figuras 9a y 9b) presentan signos de corrosión localizada. Por otro lado, los recubrimientos obtenidos en medios $\mathrm{H}_{3} \mathrm{PO}_{4}-\mathrm{NaF}$ experimentan una significativa mejoría a medida que se incrementa la concentración de ambos reactivos, observándose desde una corrosión localizada con agrietamiento severo del recubrimiento para la condición $\mathrm{H}_{3} \mathrm{PO}_{4} 1,1 \% \mathrm{v}+\mathrm{NaF}$ $0,3 \mathrm{M}$, a un recubrimiento sin signos de degradación por picadura para la condición $\mathrm{H}_{3} \mathrm{PO}_{4} 1,6 \% \mathrm{v}+\mathrm{NaF}$ $0,5 \mathrm{M}$ (Figuras $9 \mathrm{c}$ y $9 \mathrm{~d}$ ). Por otra parte, el análisis EDX de los productos de corrosión formados revela que además de presentar $\mathrm{Mg}$, existen altas cantidades de $\mathrm{O}, \mathrm{P}$, y $\mathrm{Ca}$, siendo estos elementos provenientes de la solución Hank, que al interactuar con la muestra forman probablemente fosfato de calcio (Figura 9e).

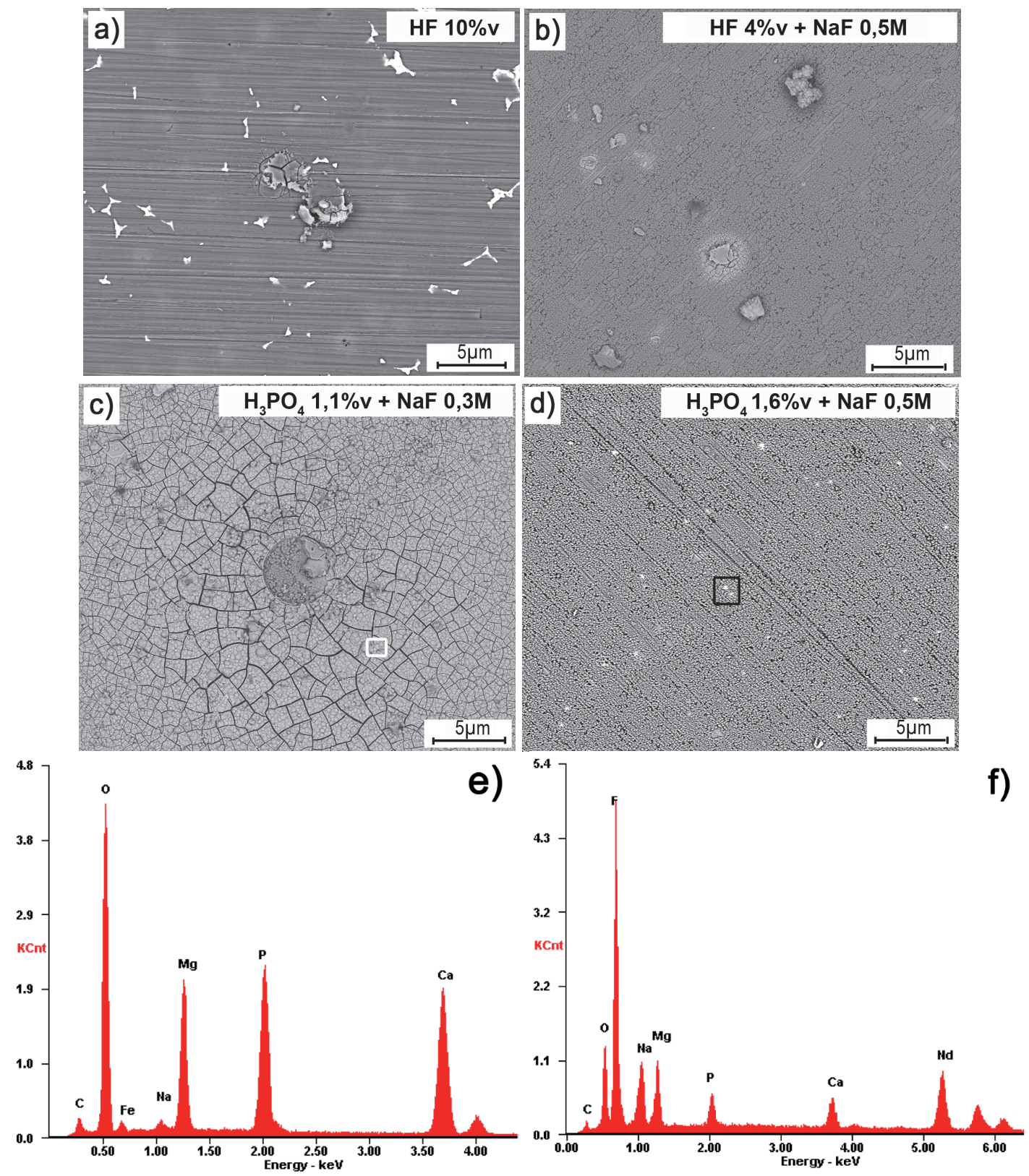

Figura 9. Micrografías electrónicas de barrido (BSE) de los recubrimientos obtenidos bajo diferentes condiciones de síntesis después de 1 día de inmersión en solución Hank: a) $\mathrm{HF} 10 \% \mathrm{v}$; b) $\mathrm{HF} 4 \% \mathrm{v}+\mathrm{NaF} 0,5 \mathrm{M}$; c) $\mathrm{H}_{3} \mathrm{PO}_{4} 1,1 \% \mathrm{v}$ $+\mathrm{NaF} 0,3 \mathrm{M}$; d) $\mathrm{H}_{3} \mathrm{PO}_{4} 1,6 \% \mathrm{v}+\mathrm{NaF} 0,5 \mathrm{M}$. e) y f) Espectros EDX de la superficie de los recubrimientos mostrados en las micrografías c) y d). 
Cabe resaltar de nuevo que para la condición $\mathrm{H}_{3} \mathrm{PO}_{4} 1,6 \% \mathrm{v}+\mathrm{NaF} 0,5 \mathrm{M}$, el espectro EDX muestra cantidades considerables de $\mathrm{F}, \mathrm{Na}$ y $\mathrm{Mg}$ (elementos representativos del recubrimiento), pero también $\mathrm{Ca}$ y $\mathrm{P}$, en menor proporción, asociado a la formación minoritaria de fosfatos de calcio y magnesio, resultado de la degradación gradual del recubrimiento (Figura 9f).

De lo anterior se comprende que el diferente mecanismo de degradación y comportamiento observadoentreambas condiciones (mediosácidos) probablemente esté atribuido al diferente grado de protección de los recubrimientos sintetizados. En el caso de los recubrimientos de hidroxifloururo de magnesio $\left(\mathrm{MgF}_{2-x}(\mathrm{OH})_{x}\right)$, algunos autores han postulado que su mecanismo de degradación está basado en un intercambio aniónico entre el ión fluoruro y las especies presentes en el medio, tales como cloruros o fosfatos, originando compuestos que dependiendo del anión puede dar lugar a especies solubles $\left(\mathrm{MgCl}_{2}\right)$ o especies insolubles (fosfatos de calcio y magnesio) [29,39].

Este intercambio es el responsable de que el recubrimiento se degrade localmente y se observe corrosión por picadura de la aleación base. Por otro lado, la presencia de una película continua de neighborita sobre la superficie del recubrimiento modifica sustancialmente su mecanismo de protección-degradación, de tal forma que la diferente naturaleza química reduce significativamente la disolución del recubrimiento de forma localizada, haciendo que su degradación sea más continua y progresiva.

\section{Conclusiones}

El empleo de soluciones de $\mathrm{H}_{3} \mathrm{PO}_{4}-\mathrm{NaF}$ permite la formación de recubrimientos doble capa, compuestos por una capa uniforme de hidroxifluoruro de magnesio $\left(\mathrm{MgF}_{2}-\mathrm{x}(\mathrm{OH}) \mathrm{x}\right)$ que crece directamente sobre el sustrato, y sobre ésta, una capa de neighborita $\left(\mathrm{NaMgF}_{3}\right)$ de morfología cúbica que crece a partir de la anterior y se hace más homogénea y compacta al incrementar la concentración de ambos reactivos.

Los recubrimientos sintetizados bajo la condición $\mathrm{H}_{3} \mathrm{PO}_{4} 1,6 \% \mathrm{v}-\mathrm{NaF} 0,5 \mathrm{M}$ presentan características protectoras de calidad superior a los obtenidos con el empleo de HF, por lo que se postulan como excelentes candidatos en términos de protección para la sustitución de este ácido, evitando los riesgos asociados a su manipulación. Este hecho se atribuyó a la presencia de una película continua de neighborita sobre la superficie del recubrimiento que modifica sustancialmente su mecanismo de protección-degradación, de tal forma que la diferente naturaleza química reduce significativamente la disolución del recubrimiento de forma localizada, haciendo que su degradación sea más uniforme y progresiva, hecho de especial interés en el diseño de recubrimientos biodegradables.

\section{Agradecimientos}

Los autores agradecen a la Vicerrectoría de Investigación y Extensión de la Universidad Industrial de Santander, Colombia (Proyecto 5456) por el apoyo financiero para el desarrollo de la investigación y a Mark Turski y Justyna Anwar de la Universidad de Manchester, Reino Unido, por el suministro de la aleación.

\section{Referencias bibliográficas}

[1] Kamachi M, Sridhar T, Raj B. Corrosion of bio implants. Sadhana. 2003;28:601-37.

[2] Witte F, Hort N, Vogt C, Cohen S, Kainer KU, Willumeit $\mathrm{R}$, et al. Degradable biomaterials based on magnesium corrosion. Curr. Opin. Solid State Mater. Sci. 2008;12:63-72.

[3] Xin Y, Hu T, Chu PK. In vitro studies of biomedical magnesium alloys in a simulated physiological environment: A review. Acta Biomater. 2011;7:1452-9.

[4] Speich M, Bousquet B, Nicolas G. Reference values for ionized, complexed, and protein-bound plasma magnesium in men and women. Clin. Chem. 1981;27:246-8.

[5] Pardo A, Merino MC, Coy AE, Viejo F, Arrabal R, Feliú S. Influence of microstructure and composition on the corrosion behaviour of $\mathrm{Mg} / \mathrm{Al}$ alloys in chloride media. Electrochim. Acta. 2008;53:7890902.

[6] Coy AE, Viejo F, Skeldon P, Thompson GE. Effect of excimer laser surface melting on the microstructure and corrosion performance of the die cast AZ91D magnesium alloy. Corros. Sci. 2010;52:387-97.

[7] Gray JE, Luan B. Protective coatings on magnesium and its alloys - a critical review. J. Alloys Compd. 2002;336:88-113.

[8] Razavi M, Fathi MH, Meratian M. Biocorrosion behavior of magnesium-fluorapatite nanocomposite for biomedical applications. 
Mater. Lett. 2010;64:2487-90.

[9] Hort N, Huang Y, Fechner D, Störmer M, Blawert $\mathrm{C}$, Witte $\mathrm{F}$, et al. Magnesium alloys as implant materials- Principles of property design for $\mathrm{Mg}-$ RE alloys. Acta Biomater. 2010;6:1714-25.

[10] Pereda MD, Alonso C, Burgos-Asperilla L, del Valle JA, Ruano OA, Perez P, et al. Corrosion inhibition of powder metallurgy $\mathrm{Mg}$ by fluoride treatmentes. Acta Biomater. 2010;6:1772-82.

[11] Kirkland NT, Lespagnol J, Birbilis N, Staiger MP. A survey of bio-corrosion rates of magnesium alloys. Corros. Sci. 2009;52:287-91.

[12] Hornberger $H$, Virtanen $S$, Boccaccini AR. Biomedical coatings on magnesium alloys - a review. Acta Biomater. 2012;8:2242-455.

[13] Kubásek J, Vojtěch D. Structural and corrosion characterization of biodegradable $\mathrm{Mg}-\mathrm{RE}$ $(\mathrm{RE}=\mathrm{Gd}, \mathrm{Y}, \mathrm{Nd})$ alloys. Trans. Nonferrous Met. Soc. China. 2013;23:1215-25.

[14] GuX, Zheng Y, Cheng Y, Zhong S, Xi T. In vitro corrosion and biocompatibility of binary magnesium alloys. Biomaterials. 2009;30:484-98.

[15] Feyerabend F, Fischer J, Holtz J, Witte F, Willumeit $\mathrm{R}$, Drücker $\mathrm{H}$, et al. Evaluation of short-term effects of rare earth and other elements used in magnesium alloys on primary cells and cell lines. ActaBiomater. 2010;6:1834-1842.

[16] Chun-yan Z, Rong-chang Z, Rong-shi C, Chenglong $L$, Jia-cheng $G$. Preparation of calcium phosphate coatings on Mg-1.0Ca alloy. Trans. Nonferrous Met. Soc. China. 2010;20:s655-59.

[17] Ng WF, Wong MH, Cheng FT. Stearic acid coating on magnesium for enhancing corrosion resistance in Hanks' solution. Surf. Coat. Technol. 2010;204:1823-30.

[18] Carboneras M, Hernández LA, Mireles YE, Hernández LS, García MC, Escudero ML. Tratamientos químicos de conversión para la protección de magnesio biodegradable en aplicaciones temporales de reparación ósea. Revista de metalurgia. 2010;46:86-92.

[19] Alvarez M, Pereda MD, del Valle JA, Fernandez M, Garcia MC, Ruano OA, et al. Corrosion behaviour of AZ31 magnesium alloy with different grain sizes in simulated biological fluids. Acta Biomater. 2010;6:1763-71.

[20] Yan T, Tan L, Xiong D, Liu X, Zhang B, Yang $\mathrm{K}$. Fluoride treatment and in vitro corrosion behavior of an AZ31B magnesium alloy. Mater. Sci. Eng. C. 2010;30:740-8.

[21] Gupta RK, Mensah-Darkwa K, Kumar D. Corrosion protective conversion coatings on magnesium disks using a hydrothermal technique. J. Mater. Sci. Technol. 2014;30:4763.

[22] Pereda MD, Alonso C, Gamero M, del Valle JA, Fernández M. Comparative study of fluoride conversion coatings formed on biodegradable powder metallurgy Mg: The effect of chlorides at physiological level. Mater. Sci. Eng. C. 2011;31:858-65.

[23] Wu L, Dong J, Ke W. Potentiostatic deposition process of fluoride conversion film on AZ31 magnesium alloy in $0.1 \mathrm{M} \mathrm{KF}$ solution. Electrochim. Acta. 2013;105:554-9.

[24] Cui X, Li Q, Li Y, Wang F, Jin G, Ding M. Microstructure and corrosion resistance of phytic acid conversion coatings for magnesium alloy. Appl. Surf. Sci. 2008;255:2098-103.

[25] Cui X, Li Y, Li Q, Jin G, Ding M, Wang F. Influence of phytic acid concentration on performance of phytic acid conversion coatings on the AZ91D alloy. Mater. Chem. Phys. 2008;111:503-7.

[26] Chiu KY, Wong MH, Cheng FT, Man HC. Characterization and corrosion studies of fluoride conversion coating on degradable $\mathrm{Mg}$ implants. Surf. Coat. Technol. 2007;202:590-8.

[27] Mertz W. The essential trace elements. Sci. 1981;213:1332-8.

[28] Carboneras M, García-Alonso MC, Escudero ML. Biodegradation kinetics of modified magnesiumbased materials in cell culture medium. Corros. Sci. 2011;53:1433-9.

[29] Zhu Y, Wu G, Zhang YH, Zhao Q. Growth and characterization of $\mathrm{Mg}(\mathrm{OH})_{2}$, film on magnesium alloy AZ31. Appl. Surf. Sci. 2011;257:6129-37.

[30] Bakhsheshi-Rad HR, Idris M, Abdul MR, Daroonparvar M. Effect of fluoride treatment on corrosion behavior of Mg-Ca binary alloy for implant application. Trans. Nonferrous Met. Soc. China. 2013;23:699-710.

[31] Conceicao TF, Scharnagl N, Blawert C, Dietzel W, Kainer KU. Surface modification of magnesium alloy AZ31 by hydrofluoric acid treatment and its effect on the corrosion behavior. Thin Solid Films. 2010;518:5209-18.

[32] Zheng RF, Liang $\mathrm{CH}$. Conversion coating treatment for AZ91 magnesium alloys by a permanganate-REMS bath. Materials and Corrosion. 2007;58:193-7.

[33] Khan AA, Marrow TJ. In-situ observation of damage mechanisms by digital image correlation during tension and low cycle fatigue of magnesium alloys. 12th International Conference on Fracture; 2009 july 12-17; Ottawa, Canada. Red Hook, NY, USA: Curran Associates, Inc; 2010.p. 871-79. 
[34]ASTM E407-07(2015), Standard Practice for Microetching Metals and Alloys, ASTM International, West Conshohocken, PA, 2015, DOI:10.1520/E0407-07R15, www.astm.org.

[35]Zhang CY, Zeng RC, Liu CL, Gao JC. Comparison of calcium phosphate coatings on $\mathrm{Mg}-\mathrm{Al}$ and $\mathrm{Mg}-\mathrm{Ca}$ alloys and their corrosion behavior in Hank's solution. Surf. Coat. Technol. 2010;204:3636-40.

[36] Norma ASTM G1-90. "Standard practice for preparing, cleaning, and evaluating corrosion test specimens". 1990.
[37]ASTM NACE / ASTMG31-12a, Standard Guide for Laboratory Immersion Corrosion Testing of Metals, ASTM International, West Conshohocken, PA, 2012, DOI: 10.1520/ G0031-12A, www.astm.org.

[38] Kielbus A. Microstructure and mechanical properties of Elektron 21 alloy after heat treatment. JAMME. 2007;20:127-30.

[39] Mao L, Yuan G, Niu J, Zong Y, Ding W. In vitro degradation behavior and biocompatibility of $\mathrm{Mg}-\mathrm{Nd}-\mathrm{Zn}-\mathrm{Zr}$ alloy by hydrofluoric acid treatment. Mater. Sci. Eng. C. 2013;33:242-50. 